\title{
Dynamic control of capillary flow in porous media by electroosmotic pumping
}

\author{
Tally Rosenfeld ${ }^{1}$ and Moran Bercovici ${ }^{1,2, *}$ \\ ${ }^{1}$ Faculty of Mechanical Engineering, Technion - Israel Institute of Technology, Haifa 3200003, ISRAEL \\ ${ }^{2}$ Department of Mechanical Engineering, The University of Texas at Austin, Austin, Texas 78712, USA \\ *Corresponding author: mberco@technion.ac.il
}

\begin{abstract}
Microfluidic paper-based analytical devices ( $\mu$ PADs) rely on capillary flow to achieve filling, mixing and delivery of liquids. We investigate the use of electroosmotic (EO) pumping as a mechanism for dynamic control of capillary flow in paper-based devices. The applied voltage can accelerate or decelerate the baseline capillary-driven velocity, as well as be used to create a tunable valve that reversibly switches the flow on and off in an electrically controlled manner. The method relies on simple fabrication and allows repeated actuation, providing a high degree of flexibility for automation of liquid delivery. We adapt the Lucas-Washburn model to account for EO pumping and provide an experimentally validated analytical model for the distance penetrated by the liquid as a function of time and the applied voltage. We show that the EO-pump can reduce filling time by 6.5-fold for channels spanning several $\mathrm{cm}$ in length, relative to capillary filling alone. We demonstrate the utilization of the EO-pump for a tunable and dynamic flow control that accelerates, decelerate and stop the flow on demand. Finally, we present the use of EO-pump for fluid flow sequencing on a paper-based device.
\end{abstract}

\section{Introduction}

The inherent ability of porous membranes, such as cellulose and nitrocellulose, to wick and transport fluids has enabled the implementation of simple and low-cost chemical and biochemical assays, including colorimetric analytical tests and lateral flow immunoassays. However, wicking alone is not sufficient for achieving sophisticated bioanalytical multi-step assays that require complex fluid and analyte handling tasks. ${ }^{1}$ Conventional enzyme-linked immunosorbent assay (ELISA), for example, require multiple individually timed steps of binding, washing and amplification, where the timing of each step is critical for the outcome of the assay. Similar automated fluidic control and sequencing are thus desired in paper-based assays. $^{2,3}$

The simplest way to control the flow rate and delivery time of reagents is by varying the geometry of the channels such as their length and width. ${ }^{4,5}$ Alternatively, fluid flow can be delayed via manipulation of capillary flow in porous media, including through the parameters of medium pore size ${ }^{6}$, contact angle ${ }^{7}$, or fluid viscosity ${ }^{8}$. Other approaches involve altering the channel wettability by using ultraviolet light treatment ${ }^{9}$ or depositing material to the channels, such as wax ${ }^{10}$. Complete on/off valving has also been extensively explored for paper-based microfluidic devices. For example, wettability-switching valves render hydrophobic surfaces into hydrophilic by an electrical plasma ${ }^{11}$ or by electrowetting ${ }^{12-14}$. The biphilic nature of surfactants can also be exploited to bridge a hydrophobic gap in the flow path. ${ }^{15,16}$ The main class of paper fluidic valves, however, is mechanically actuated valves in which the contact between 
two channels is controlled, ${ }^{17}$ either via the operator or automated via an element responsive to a particular external stimulus. Many approaches have been reported for mechanically actuated valves including pushbuttons $^{18}$, folding ${ }^{3,19}$, sliding component ${ }^{20}$ and magneto-mechanical ${ }^{17,21}$.

Each approach has its own advantages and disadvantages, including complexity of fabrication, automation, functionality and cost. For example, while electrowetting-based methods are easy to control electronically, they are irreversible and allow only one-time switching. On the other hand, magneto-mechanical valves are reversible but require multiple components and precise assembly. In term of functionality, reversible valves are limited to turning the flow on and off but cannot then control the flow rate which remains governed by capillary wicking.

In this work we explore the use of electroosmotic (EO) pumping as a mechanism to accelerate, decelerate, or stop capillary flow in paper-based devices using electric actuation. EO-pumps use electroosmosis to drive liquids and generate both flow rate and significant pressure capacity through pores or channels. ${ }^{22}$ Although electroosmosis first described by Reuss in $1809,{ }^{23}$ it was first utilized in an analytical method in 1974 when Pretorius et al. ${ }^{24}$ used it to drive a liquid chromatographic separation. Abelev and Karamova implemented EO-pumping on cellulose acetate and nitrocellulose porous membranes, as a mechanism for driving immunoreactions. ${ }^{25,26}$ In recent years, EO-pumps receive increasing attention in microfluidics since they have several unique features compared to other micropumps: they generate constant pulse-free flows, their flow magnitude and direction are convenient to control, they are readily integrated with lab-on-chip devices, and they have no moving parts. ${ }^{27-32}$

In contrast to previous work that utilized EO-flow (EOF) to drive flow in already wet membranes, we here investigate the use of EO pumping for dynamic control of capillary flows during the filling process in paperbased devices. We present an analytical model for the distance penetrated by the liquid in porous media affected by the application of an integrated EO-pump and characterized the deviation from LucasWashburn flow. We then provide the experimental results of the penetration distance and filling time in porous media, validating the theory. We further show how the device can be utilized for tunable flow control using EO acceleration or EO deceleration, and finally demonstrate fluid flow sequencing on paper-based device. This method relies on simple electrodes that can be easily fabricated and repeatedly actuated, allowing high flexibility in automation and sequencing for capillary filling processes.

\section{Operation principle and theory}

We here utilize the common approach for analysis of porous media wherein the substrate is modeled as a bundle of independent, parallel, tortious, idealized cylindrical capillaries (pores), which have equal radii, $a$, and an equal value of zeta potential, $\zeta{ }^{22}$ We model the flow within one capillary, and then extend it to the entire porous media. Figure 1 presents a schematic illustration of one such capillary connected to a reservoir at one end and subjected to a DC voltage difference between two electrodes - one in the reservoir and one in the channel. When liquid is placed into the reservoir, it initially fills the capillary by capillary force alone, until it reaches the in-channel electrode at time $t_{p}$ located at $l_{p}$. As the liquid wets the in-channel electrode, the electric circuit is closed, resulting in EOF and pressure driven flow in zone 1 of the channel. The liquid then continues filling the channel under the combined effect of capillary pressure and pressure driven flow. We seek to determine the penetration distance as a function of time, $l(t)$. 


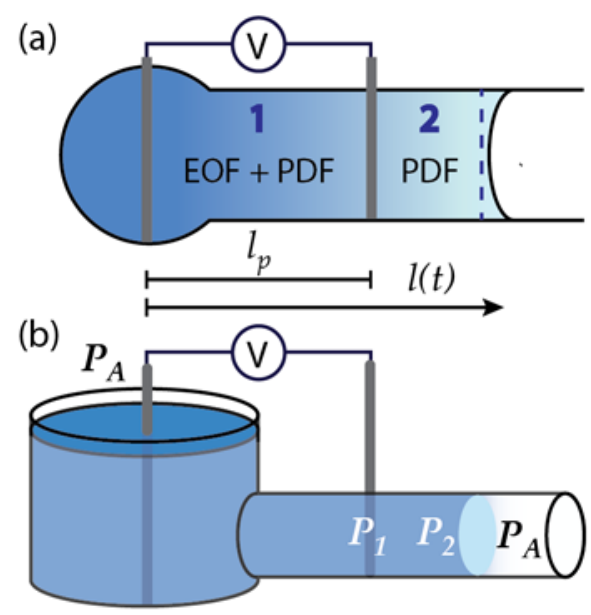

Figure 1. Schematic (a) top-view and (b) side-view illustrations of the problem formulation, showing liquid penetrating into a single capillary under the combined influence of capillary force and EOF. As the liquid wets the in-channel electrode, voltage, $V$, is applied between two electrodes - one in the reservoir and one in the channel, at a distance $l_{p}$, establishing an EO-pump. The discontinuity in EOF induces a pressure $P_{1}$ over the in-channel electrode. In zone 1 (between the electrodes) the flow is driven by EOF and the pressure difference between $P_{1}$ and the reservoir's atmospheric pressure, $P_{A}$, resulting in pressure driven flow (PDF). In zone 2 (between the in-channel electrode and the meniscus) the flow is driven by the pressure difference between $P_{1}$ and the capillary pressure. We denote the distance penetrated by the liquid at time $t$ as $l(t)$.

Assuming a small circular cross-section capillary with a high axial-length-to-radius ratio, the velocity profile of liquid in zone 1 of the channel moving under the influence of both an axial voltage and pressure gradient is given $b^{22}$

$$
u(r)=-\frac{a^{2} P_{z}}{4 \eta}\left(1-\frac{r^{2}}{a^{2}}\right)-\frac{\varepsilon \zeta E_{p}}{\eta}\left(1-\frac{\varphi(r)}{\zeta}\right),
$$

where $a$ is the capillary radius, $P_{z}=\Delta P / l(t)$ is the streamwise pressure gradient, $\eta$ is the liquid viscosity, $\varepsilon$ is the permittivity of the liquid, $\zeta$ is the zeta potential of the surface of the capillary, $E_{p}=V / l_{p}$ is the applied electric field and $\varphi(r)$ is the potential distribution between the surface and the bulk due to the electric double layer (EDL). The volumetric flow rate in zone 1 is thus,

$$
Q_{1}(t)=-A \cdot\left[\frac{a^{2}}{8 \eta} \frac{\left(P_{1}-P_{A}\right)}{l_{p}}+\frac{\varepsilon \zeta E_{p}}{\eta} f\right]
$$

where $A$ is the cross-sectional area, $P_{A}$ is the atmospheric pressure, $P_{1}$ is the pressure around the in-channel electrode resulting from the discontinuity in the EOF and $f$ is a constant resulting from the integration over the electric field in the EDL. Under the Debye-Hückel approximation $f$ takes the form

$$
f \cong \int_{0}^{a}\left(1-\frac{I_{0}\left(r / \lambda_{D}\right)}{I_{0}\left(a / \lambda_{D}\right)}\right) \frac{2 r}{a^{2}} d r=1-\frac{2 I_{1}\left(a / \lambda_{D}\right)}{\left(a / \lambda_{D}\right) I_{0}\left(a / \lambda_{D}\right)}
$$


where $I_{0}$ and $I_{1}$ are the zero- and first-order modified Bessel function of the first kind, respectively, and $\lambda_{D}$ is the Debye length. For a thin electrical double layer, $f$ approaches 1.

In zone 2, the flow is driven purely by pressure, and the flow rate is given by

$$
Q_{2}(t)=-A \cdot \frac{\left(P_{2}-P_{1}\right)}{\left(l(t)-l_{p}\right)} \frac{a^{2}}{8 \eta},
$$

where $P_{2}$ is the capillary pressure. We model the capillary pressure using the Young-Laplace equation ,

$$
P_{2}=P_{A}-\frac{2 \gamma \cos \theta}{a}
$$

where $\gamma$ is the surface tension of the liquid, and $\theta$ is the contact angle, which we assume is independent of the applied voltage, $V . P_{1}$ is obtained by requiring mass-flow conservation between zones 1 and 2 ,

$$
P_{1}=P_{2} \frac{l_{p}}{l(t)}+P_{A}\left(1-\frac{l_{p}}{l(t)}\right)-\frac{8 \eta l_{p}}{a^{2}}\left(1-\frac{l_{p}}{l(t)}\right) \frac{\varepsilon \zeta E_{p}}{\eta} f .
$$

Substituting eq.(4) and eq.(5) into eq.(2), and following Washburn ${ }^{33}$ substituting the average velocity, $Q(t) / A$, for the rate of penetration, $d l(t) / d t$, we obtain a closed-form equation for the penetration distance,

$$
\frac{d l(t)}{d t}=\frac{a^{2}}{8 \eta} \frac{l_{p}}{l(t)}\left[-\frac{8 \eta}{a^{2}} \frac{\varepsilon \zeta E_{p}}{\eta} f+\frac{2 \gamma \cos \theta}{a l_{p}}\right] .
$$

Solving the ODE, and substituting $E_{p}=V / l_{p}$ we obtain the final expression for the penetrated distance squared, $l^{2}(t)$, in a single capillary

$$
l^{2}(t)=\left(\frac{2 \varepsilon \zeta V}{\eta} f+\frac{a \gamma \cos \theta}{2 \eta}\right) t-\frac{2 \varepsilon \zeta V}{\eta} f t_{p} .
$$

Importantly, for the case without the EO-pump ( $V=0)$, eq.(7) reduces to the Lucas-Washburn equation $^{33,34}$,

$$
l^{2}(t)=\frac{a \gamma \cos \theta}{2 \eta} t
$$

which describes the penetration distance for $t<t_{p}$. Using the definition of tortuosity (interconnectedness), $\tau=l^{2} / L^{2}$, where the average capillary length is denoted by $l$, and the physical length of the porous media (external dimension) is denoted by $L$, we can extend the penetrated distance squared from a single capillary to the entire porous media, 


$$
L^{2}(t)=\left\{\begin{array}{cc}
\frac{a \gamma}{2 \eta} \frac{\cos \theta}{\tau} t & t<t_{p} \\
\left(\frac{2 \varepsilon V}{\eta} \frac{\zeta}{\tau} f+\frac{a \gamma}{2 \eta} \frac{\cos \theta}{\tau}\right) t-\frac{2 \varepsilon V}{\eta} \frac{\zeta}{\tau} f t_{p} & t \geq t_{p}
\end{array}\right.
$$

This result shows that the distance penetrated by the liquid, affected by an EO-pump, is directly proportional to the square root of time, is independent on the length of the pump, and is directly dependent on the applied voltage. Importantly, negative voltage could be applied (i.e. an electric field in the opposite direction) for delaying or even stopping the capillary flow in the porous channel.

\section{Materials and methods}

\section{1. $\mu$ PAD fabrication and device assembly}

In all experiments, we used wax printing-based fabrication ${ }^{35-37}$ on a nitrocellulose membrane $(0.45 \mu \mathrm{m}$ pores, BA85 Protran, $300 \mathrm{~mm} \times 4 \mathrm{~m}$, Whatman, GE Healthcare), which is $\sim 100 \mu \mathrm{m}$ thick, and was refer to here as "paper”. We designed the paper-chip’s geometry in Autodesk AutoCAD 2013 (Autodesk Inc, San Rafael, CA), and fabricated the microfluidic paper-chip by printing (ColorQube 8580DN, Xerox Corporation, Norwalk, CT) the channels' side walls on one side of a membrane, pre-cut to an A6 size. To avoid paper jamming due to electrostatic adhesion of the nitrocellulose in the printing path, before printing we placed the membrane on top of a filter paper covering its entire area and facing away from the print side. To then melt the wax and have it penetrated the depth of the channel, we passed it through a temperature-controlled lamination machine (335 R6, SKYDBS Co., Seoul, Korea) four times at $110^{\circ} \mathrm{C}$ and a feeding speed of $150 \mathrm{~mm} / \mathrm{min}$. Before the heating process, we covered the paper with an additional layer of filter paper on top and inserted them into a plastic lamination pouch that protected the papers from wrapping around the rollers. We then placed on each paper-chip two electrodes made of $\sim 2 \mathrm{~mm}$ wide strips of aluminum foil tape (type 431, 3M, St. Paul, MN). Finally, we used transparent masking tape (type 8911, $3 \mathrm{M}$, St. Paul, MN) to seal the channels from the top and to cover the entire paper-chip from the bottom. We kept the paper-chips in a closed chamber containing a small open water tube intended to ensure sufficient humidity, preventing the membranes from becoming increasingly hydrophobic under dry conditions.

We used two paper-chip designs: (i) In sections 4.1 and 4.2, four independent parallel channels were used, connected on both ends to common reservoirs. The nominal paper channel dimensions were $21 \mathrm{~mm}$ length and $2.5 \mathrm{~mm}$ width. (ii) In section 4.3, a Y-shaped channel design was used to demonstrate fluid flow sequencing on paper. In all experiments, the electrodes were connected to a high voltage sourcemeter (model 2410, Keithley Instruments, Cleveland, $\mathrm{OH}$ ) by two electrical leads. We placed one electrode in the reservoir, and the other in the channel - $2.5 \mathrm{~mm}$ from its entrance. As illustrated in Figure 2, the paper-chips were held between two plates of Perspex with a silicone rubber sealing layer between the chip and top plate. To illuminate the paper from below, we placed a transparent rectangular Perspex in the middle of the bottom Perspex layer, right beneath the channels area (not shown in figure). The two Perspex layers were held together by four screws. The common reservoirs on both ends were kept open to the atmosphere. 




Figure 2. Schematic illustration of the paper-chip used in sections 4.1 and 4.2 and the device assembly. The paper-chip was supported by a rigid layer of Perspex containing a $5 \times 2 \mathrm{~cm}$ transparent Perspex plate at its center to allow for diascopic illumination. The electrodes were made of $\sim 2 \mathrm{~mm}$ thick strips of aluminum tape connected to high voltage sourcemeter by two electrical leads. On top of the chip was a silicone rubber sealing layer and an additional transparent and rigid Perspex plate. The entire structure was held together using fours screws connecting the top and bottom plates.

\subsection{Experimental setup}

For the experiments described in sections 4.1 and 4.2, we used an upright microscope (AZ100 multizoom, Nikon, Tokyo, Japan) equipped with a $1 \mathrm{X}$ objective (NA = 0.1, WD = 35 mm, AZ-Plan Apo, Nikon, Tokyo, Japan) and a light source (C-FI230, Nikon, Tokyo, Japan). We illuminated the paper-chip from beneath (diascopic illumination), which gave a good contrast between dry and wet areas and allowed to identify the liquid front easily. Images were captured using a 16 bit, 2048 x 2048-pixel array sCMOS camera (Zyla 4.2 plus, Andor, Belfast, Ireland) cooled to $0^{\circ} \mathrm{C}$. Images described in section 4.3 were captured by an iPhone 7 plus camera. For all experiments we used a high voltage sourcemeter (model 2410, Keithley Instruments, Cleveland, $\mathrm{OH})$.

We began all experiments by placing a paper-chip inside the device, attaching the electrical leads to the aluminum strips, filling the right reservoir with $150 \mu \mathrm{l}$ of the relevant buffer solution and finally applying constant voltage. Once the liquid front came in contact with the inner electrode inside the channel, the electrical circuit was closed and initiated EOF (the electrode in the reservoir served as ground). The direction of the electric field determined whether EO acceleration or EO deceleration was applied.

\subsection{Choice of experiments conditions}

The buffer solutions we used for the experiments in section 4.1 were $10 \mathrm{mM}$ and $100 \mathrm{mM}$ Tris-HCl at $\mathrm{pH} 8$. For all other experiments we used $10 \mathrm{mM}$ Tricine and $20 \mathrm{mM}$ Bistris as the buffer solution (pH 7.4). The $\mathrm{pH}$ values of the buffers were selected to fit the typical range used in biological applications. Tricine, Trizma and Bistris were obtained from Sigma-Aldrich (St. Louis, MO) and HCl was obtained from Merck (Darmstadt, Germany). All solutions were made using DI from a Millipore Milli-Q system (Billerica, MA).

For the demonstration of fluid flow sequencing on $\mu \mathrm{PAD}$, we used three colors: red and blue were food coloring dyes (Maimon's, Beersheba, Israel) and fluorescein sodium salt (Sigma-Aldrich, St. Louis, MO) served as the yellow color. 


\section{Results and discussion}

\subsection{Effect of EO pumping on penetration distance and filling time in porous media}

Figure 3b presents experimental measurements of the penetrated distance (squared, $L^{2}$ ) vs time, for various EO-pump voltages $(0,100,150$ and $200 \mathrm{~V})$. The solid lines represent the linear least squares fit to $L^{2}$, expressed by eq.(9) where we assumed $f=1$ since our nitrocellulose membrane has a pore diameter of approximately $0.45 \mu \mathrm{m}$, and debye length of $3 \mathrm{~nm}$ (estimated for $10 \mathrm{mM}$ buffer).

We fitted all the data points from all experimental simultaneously, with $\cos \theta / \tau$ and $\zeta / \tau$ serving as the free fitting parameters (all fits performed for times $t>t_{p}$ ). Assuming standard properties for water at $25^{\circ} \mathrm{C}$ $\varepsilon=7 \cdot 10^{-10} \mathrm{C} \mathrm{V}^{-1} \mathrm{~m}^{-1}, \gamma=72 \cdot 10^{-3} \mathrm{~N} \mathrm{~m}^{-1}$ and $\eta=8.9 \cdot 10^{-4} \mathrm{~N} \mathrm{sec} \mathrm{m}^{-2}$, the values for $\cos \theta / \tau$ and $\zeta / \tau$ were found to be 0.093 and $-0.02 \mathrm{~V}$, respectively. A tortuosity value of $\tau \sim 1.9$, as measured for $0.45 \mu \mathrm{m}$ diameter nitrocellulose, ${ }^{38}$ yields $80^{\circ}$ and $-45 \mathrm{mV}$ for $\theta$ and $\zeta$, respectively. These values are well within expected and common ranges for contact angle and surface potential on paper. ${ }^{39,40}$

A different presentation of the same experimental data is shown in Figure 3a, where the time required for the liquid to penetrate a set of fixed distances, $L=1.05,1.8$ and $2.55 \mathrm{~cm}$, is presented against the applied voltage. The dashed lines represent the theoretical value for the filling time, $t$, as a function of the applied voltage, for the different lengths, as obtained by extracting the time from eq.(9),

$$
t(L, V)=\left\{\begin{array}{cc}
\frac{2 \eta}{a \gamma}\left(\frac{\cos \theta}{\tau}\right)^{-1} L^{2} & t<t_{p} \\
\left(L^{2}+\frac{2 \varepsilon V}{\eta} \frac{\zeta}{\tau} t_{p}\right)\left(\frac{2 \varepsilon V}{\eta} \frac{\zeta}{\tau}+\frac{a \gamma}{2 \eta} \frac{\cos \theta}{\tau}\right)^{-1} & t \geq t_{p} .
\end{array} .\right.
$$

The curves described by eq.(10) provide a good prediction for the temporal behavior of the filling process as well as of the effect of the pump's voltage on the rate of filling, thus supporting the baseline assumptions for the model.

Figure 3 shows that activating the EO-pump at $200 \mathrm{~V}$ decreases the capillary filling time of $2.55 \mathrm{~cm}$ by 6.5-fold. As indicated by eq.(10), for sufficiently long filling distances, the relative gain from the pump actuation is given by $\frac{4 \varepsilon V}{a \gamma} \frac{\zeta}{\tau}\left(\frac{\cos \theta}{\tau}\right)^{-1}+1$, which for our experimental conditions at $200 \mathrm{~V}$ yields a 10 -fold gain.

The upper bound on the applied voltage is set by electrolysis effects on the in-channel electrode, causing both bubbles and $\mathrm{pH}$ changes in the pump section of the channel. At higher voltages, we observed irregular electric current patterns and loss of repeatability. To test which of the effects is more dominant we repeated the same set of experiments with a higher ionic strength buffer (100 mM, see SI Figure S1) and obtained a similar 200V bound, though with lower $\mathrm{R}^{2}$ fitting quality to the model. Since the electric current at this higher ionic strength was 10 -fold higher, driving a proportionally higher rate of gas production, we conclude that the limits of the system are set by its buffering capacity (i.e. $\mathrm{pH}$ changes) rather than blockage by bubbles. 


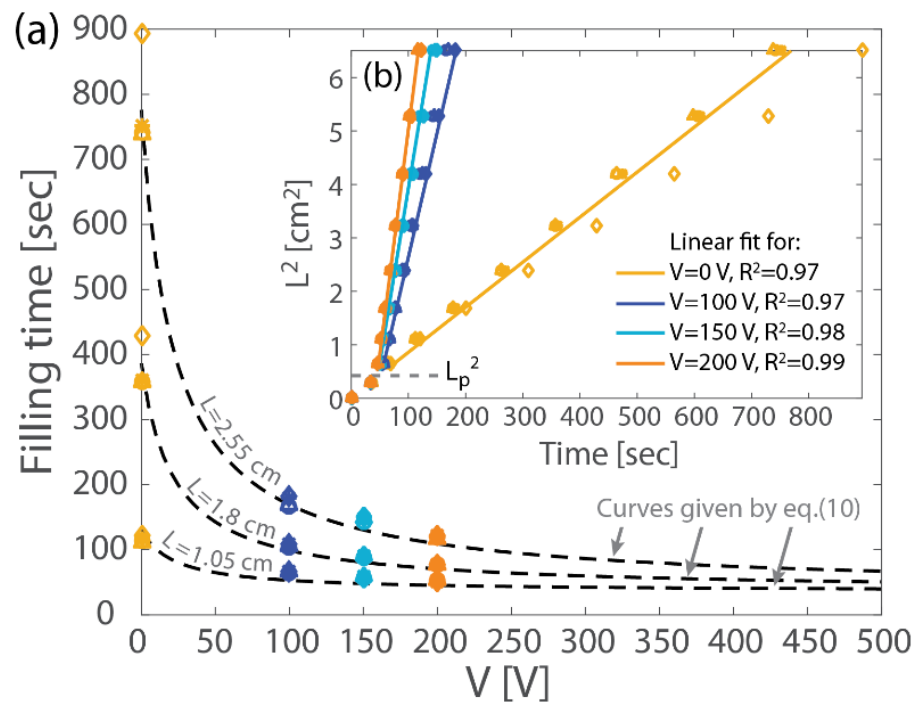

Figure 3. Experimental results and their fitting to the analytical model for capillary filling in porous media with and without EO pumping. (a) Experimental results (four repeats) showing the filling time vs. applied EO-pump voltage for three different penetration distances ( $L=1.05,1.8$ and $2.55 \mathrm{~cm}$ ). Each marker shape represents an individual experiment, and the dashed lines represent the theoretical times obtained by eq.(10). (b) The same experimental results showing penetration distance squared, $L^{2}$, vs. time. The EOpump zone length is indicated as $L_{p}{ }^{2}$. Each marker shape represents an individual experiment, and the solid lines represent the linear least squares fit to $L^{2}$, expressed by eq.(9), with $\cos \theta / \tau$ and $\zeta / \tau$ serving as the free fitting parameters. Results show that the EO-pump can significantly shorten the filling time to a given distance, with greater gain for longer filling distances. The buffer solution is $10 \mathrm{mM}$ Tris-HCl.

\subsection{Valving and flow rate control using EO pumping}

The EO-pump can be used not only to create a supporting pressure gradient for accelerating capillary filling, but also to establish an adverse pressure gradient to slow down or hold the capillary front stationary. Figure 4 presents a side-by-side comparison of the location of the capillary front in a channel containing an EO-pump (blue lines) and in a standard control channel (orange lines, governed by Lucas-Washburn dynamics). The EO-pump is initially turned off, and the capillary fronts in both channels proceed at the same rate. At $\mathrm{t}=80 \mathrm{~s}$, we apply a negative voltage of $-250 \mathrm{~V}$ to create a pressure gradient opposing capillary filling, and suspend the flow for 2 min. We then turn off the pump and observe again standard capillary filling in both channels, with the capillary-front in the control channel leading. At $t=320 \mathrm{~s}$, we apply a supporting pressure gradient using $200 \mathrm{~V}$, and observe the capillary front in the EO-pump channel rapidly overtaking the front in the control channel. We then again turn off the pump, and observe again standard capillary filling, this time with the front in the EO-pump channel leading. This illustrates the use of the EOpump in porous media not only as a mechanism for accelerated filling, but also as a flow control element, allowing dynamic valving with the ability to accelerate, delay, or stop the flow. 




Figure 4. Experimental demonstration of the use of EO pumping for acceleration or deceleration of capillary flow in paper-based devices. Each curve (with four repeats) presents the penetration distance vs time of an individual experiment, with blue asterisks corresponding to an EO-pump controlled channel and orange circles corresponding to a control channel with no active elements. The top panel presents the voltage applied to the pump as a function of time. (a) When no voltage is applied, the capillary front follows the Lucas-Washburn equation in both channels. (b) Applying a negative voltage results in an adverse pressure gradient which opposes the capillary force and suspends the flow for a desired amount of time (here $120 \mathrm{~s}$ ). (c) When the voltage is switched off, normal capillary filling continues, with the capillary front in the control channel leading by approximately $0.5 \mathrm{~cm}$. (d) Applying a positive voltage for $60 \mathrm{~s}$ significantly accelerates the penetration rate, allowing the capillary front in the EO-pump channel to overtake the front in the control channel. (e) When we again switch off the voltage, capillary filling proceeds, this time with the front in the EO-pump channel leading by approximately $0.5 \mathrm{~cm}$.

\subsection{Fluid flow sequencing demonstration on paper-based device}

Figure 5 presents a test device consisting of three channels leading to a common mixing region at the center. Each of the channels is connected on its other end to a separate reservoir. The two $1 \mathrm{~cm}$-long channels shown on the right contain EO-pumps, whereas the $0.8 \mathrm{~cm}$-long channel on the left has no control elements. We spotted a small region of colored dye in each of the channels, outside of the EO-pump region. In this way, the electric mobility of the dye does not take a role in its migration. Any $\mathrm{pH}$ changes are also limited to the pump region and do no propagate further downstream.

We applied $200 \mathrm{~V}$ to the blue channel and $-250 \mathrm{~V}$ to the red channel and filled all three reservoirs simultaneously with the buffer solution. At first, the three liquid fronts proceeded simultaneously under pure capillary flow, until the liquid reached the in-channel electrodes thus closing the electric circuit and initiating EOF. As a result, filling of the channel containing the blue dye was accelerated while the flow in the channel containing the red dye was halted in place. After $55 \mathrm{~s}$, when the blue dye arrived at the end of its channel, we switched the directions of the electric fields, i.e. applied $-250 \mathrm{~V}$ to hold the blue liquid front in place, and $200 \mathrm{~V}$ to accelerate the filling of the red-dye channel. At $\mathrm{t}=100 \mathrm{~s}$, when the red liquid front arrived at the center, we switched the direction of the electric field (i.e. applied -250 V) at the red-dye channel again to hold both the blue and red fronts in place. The yellow liquid was flowing under capillary 
pressure alone from the beginning until $\mathrm{t}=250 \mathrm{~s}$, when it finally arrived at the center and connected all three channels. Integration of this tool for liquid manipulation on paper-based devices can be used to reduce assay time and to perform simple automatic sequential delivery of sample, conjugates, reagents, wash and rinse solutions etc.


Figure 5. Time-lapse schematics (left) and experimental images (right) demonstrating the use of EO pumping for sequential delivery of liquids on a paper-based device. (a) The three reservoirs are simultaneously filled with liquid, which advances into the channels under capillary flow. (b) The electric field in the blue-dye channel is configured to accelerate the filling, whereas in the red-dye channel it is set to oppose the capillary pressure and hold the liquid in place. The channel containing the yellow dye lacks an EO-pump and thus follows standard capillary filling. (c) when the blue liquid front arrived at the junction, we switched the directions of the electric fields to stop the blue liquid and accelerate the red one. (d) When the red liquid arrived at the junction, we set both channels to halt. The yellow liquid continued advancing under capillary pressure until it reached the junction and connected the three streams.

\section{Conclusions}

In this paper we demonstrated the use of EO-pump on $\mu \mathrm{PAD}$, for controlling capillary filling by either delaying or accelerating the filling. The analytical model that we provided for the filling distance in porous media enables prediction of the filling time required for a given applied voltage and a desired filling distance. We validated the model experimentally and showed that the EO-pump can reduce filling time by 6.5-fold for channels spanning several $\mathrm{cm}$ in length. Finally, we showed that using EO-pump, temporal control of filling rate can be achieved, enabling programmable sample delivery on paper-based devices.

Supplementing capillary flow in paper with EO pumping has several key advantages: it allows dynamic control of the capillary flow enabling both valving and flow rate control, it is operated electronically and 
thus can be automated, the valve can be used reversibly (opened and closed) multiple times, it requires little power for operation (100 $\mathrm{mW}$ or less) and is simple to fabricate. However, it requires the use of high voltages (hundreds of volts) and can only be actuated once the liquid in the channel connects the two electrodes. Most importantly, the performance of the pump strongly depends on the electrolyte composition of the liquid and may vary between applications. $\mathrm{pH}$ and ionic strength are likely the most important parameters to control, as they directly affect the zeta potential. While for biological purposes, neutral solutions are typically favorable, the EO-pump would benefit from higher $\mathrm{pH}$ values for which the membranes are more significantly deprotonated. ${ }^{41}$

Clearly, the strength of the EO-pump is directly proportional to the applied voltage, and faster filling time could be achieved with higher voltages. However, if the applied voltage is too high, the high electrical current will cause significant hydrolysis and bubble formation which increase the resistance and can ultimately block the current. The creation of bubbles also increases run-to-run variability. One potential approach to reduce bubble formation is to use electrodes based on an $\mathrm{Ag} / \mathrm{AgCl}$ redox reaction. ${ }^{42}$ However, this may be less effective at the high voltages used in such EO-pumps systems, and one must take into consideration the migration of silver-chloride, chloride and silver ions into the system. Alternatively, in order to eliminate bubbles formation, alternating current (AC) EO-pumps (or ACEOPs) may be considered. ${ }^{28}$

In this work we focused on demonstrating the feasibility of operation of a simple and low-cost device and used flat aluminum foil electrodes for operating the EO-pump. Aluminum is highly conductive, readily available and inexpensive, yet oxidizes fairly quickly. For run times on the orders of minutes, as we had in the work, we did not see adverse effects to the use of aluminum. However, for higher voltages or longer running time, electrodes made of more inert materials may be necessary.

Since the power consumption of the pump is low, its operation from a small battery source is feasible as has been demonstrated in other electrokinetic applications. ${ }^{43,44}$ Several methods for electrode deposition on paper-based devices have also been demonstrated. ${ }^{45}$ Integration of EO-pumps for liquid manipulation can potentially enhance the functionality of paper-based devices, reducing assay time, and enabling simple automatic sequential delivery of biological samples, conjugates, reagents, and wash solutions. Furthermore, once the paper is entirely wet, EO-pumping provides a mean to continue flowing liquid as previously shown by Abelev and Karamova ${ }^{25,26}$.

\section{Acknowledgments}

We thank Ran Eshel and Baruch Rofman for useful discussions and for designing the device apparatus. This project has received funding from the European Research Council (ERC) under the European Union's Horizon 2020 Research and Innovation Programme, grant agreement no. 678734 (MetamorphChip). T.R. is grateful to the Azrieli Foundation for the award of an Azrieli Fellowship.

\section{References}

1 M. M. Gong and D. Sinton, Chem. Rev., 2017, 117, 8447-8480.

2 S. Ramachandran, E. Fu, B. Lutz and P. Yager, Analyst, 2014, 139, 1456-1462.

3 A. C. Glavan, D. C. Christodouleas, B. Mosadegh, H. D. Yu, B. S. Smith, J. Lessing, M. T. FernándezAbedul and G. M. Whitesides, Anal. Chem., 2014, 86, 11999-12007.

4 E. Fu, B. Lutz, P. Kauffman and P. Yager, Lab. Chip, 2010, 10, 918-920.

5 G. E. Fridley, H. Le and P. Yager, Anal. Chem., 2014, 86, 6447-6453. 
6 J. H. Shin, J. Park, S. H. Kim and J.-K. Park, Biomicrofluidics, 2014, 8, 054121.

7 H. Noh and S. T. Phillips, Anal. Chem., 2010, 82, 8071-8078.

8 B. Lutz, T. Liang, E. Fu, S. Ramachandran, P. Kauffman and P. Yager, Lab. Chip, 2013, 13, 2840 2847.

9 J. Songok and M. Toivakka, Microfluid. Nanofluidics, 2016, 20, 63.

10 H. Noh and S. T. Phillips, Anal. Chem., 2010, 82, 4181-4187.

11 Y. Jiang, Z. Hao, Q. He and H. Chen, RSC Adv., 2016, 6, 2888-2894.

12 A. Ainla, M. M. Hamedi, F. Güder and G. M. Whitesides, Adv. Mater., 2017, 29, 1702894.

13 C. K. W. Koo, F. He and S. R. Nugen, Analyst, 2013, 138, 4998-5004.

14 F. He, J. Grimes, S. D. Alcaine and S. R. Nugen, Analyst, 2014, 139, 3002-3008.

15 H. Chen, J. Cogswell, C. Anagnostopoulos and M. Faghri, Lab. Chip, 2012, 12, 2909-2913.

16 R. Gerbers, W. Foellscher, H. Chen, C. Anagnostopoulos and M. Faghri, Lab. Chip, 2014, 14, 40424049.

17 M. Fratzl, B. S. Chang, S. Oyola-Reynoso, G. Blaire, S. Delshadi, T. Devillers, T. Ward, N. M.

Dempsey, J.-F. Bloch and M. M. Thuo, ACS Omega, 2018, 3, 2049-2057.

18 A. W. Martinez, S. T. Phillips, Z. Nie, C.-M. Cheng, E. Carrilho, B. J. Wiley and G. M. Whitesides, Lab. Chip, 2010, 10, 2499-2504.

19 J. Ding, B. Li, L. Chen and W. Qin, Angew. Chem. Int. Ed., 2016, 55, 13033-13037.

20 J. T. Connelly, J. P. Rolland and G. M. Whitesides, Anal. Chem., 2015, 87, 7595-7601.

21 X. Li, P. Zwanenburg and X. Liu, Lab. Chip, 2013, 13, 2609-2614.

22 S. Yao and J. G. Santiago, J. Colloid Interface Sci., 2003, 268, 133-142.

23 F. F. Reuss, Mem Soc Imp Nat. Moscou, 1809, 2, 327-337.

24 V. Pretorius, B. J. Hopkins and J. D. Schieke, J. Chromatogr. A, 1974, 99, $23-30$.

25 G. I. Abelev and E. R. Karamova, Anal. Biochem., 1984, 142, 437-444.

26 G. I. Abelev and E. R. Karamova, Mol. Immunol., 1989, 26, 41-47.

27 X. Wang, S. Wang, B. Gendhar, C. Cheng, C. K. Byun, G. Li, M. Zhao, S. Liu, C. K. Byun and M. Zhao, TrAC Trends Anal. Chem., 2009, 28, 64-74.

28 X. Wang, C. Cheng, S. Wang and S. Liu, Microfluid. Nanofluidics, 2009, 6, 145-162.

29 X. Fu, N. Mavrogiannis, S. Doria and Z. Gagnon, Lab. Chip, 2015, 15, 3600-3608.

30 N. K. Ranjit and G. C. Shit, Energy, 2017, 128, 649-660.

31 X. Li, S. Liu, P. Fan, C. F. Werner, K. Miyamoto and T. Yoshinobu, Sens. Actuators B Chem., 2017, 248, 993-997.

32 Y. N. Wang and L. M. Fu, Microelectron. Eng., 2018, 195, 121-138.

33 E. W. Washburn, Phys. Rev., 1921, 17, 273.

34 R. Lucas, Kolloid-Z., 1918, 23, 15-22.

35 E. Carrilho, A. W. Martinez and G. M. Whitesides, Anal. Chem., 2009, 81, 7091-7095.

36 Y. Lu, W. Shi, L. Jiang, J. Qin and B. Lin, ELECTROPHORESIS, 2009, 30, 1497-1500.

37 Y. Lu, W. Shi, J. Qin and B. Lin, Anal. Chem., 2010, 82, 329-335.

38 A. Shrestha, University of Colorado, 2012.

39 S. Mendez, E. M. Fenton, G. R. Gallegos, D. N. Petsev, S. S. Sibbett, H. A. Stone, Y. Zhang and G. P. López, Langmuir, 2010, 26, 1380-1385.

40 W. R. Bowen and R. A. Clark, J. Colloid Interface Sci., 1984, 97, 401-409.

41 H. J. Lee, J. H. Choi, J. Cho and S. H. Moon, J. Membr. Sci., 2002, 203, 115-126.

42 P. Vulto, P. Kuhn and G. A. Urban, Lab. Chip, 2013, 13, 2931.

43 B. Y. Moghadam, K. T. Connelly and J. D. Posner, Anal. Chem., 2014, 86, 5829-5837.

44 X. Li, L. Luo and R. M. Crooks, Lab. Chip, 2015, 15, 4090-4098.

45 C. Silveira, T. Monteiro, M. Almeida, C. M. Silveira, T. Monteiro and M. G. Almeida, Biosensors, 2016, 6, 51. 